\title{
Aislamiento acústico a ruido aéreo en acristalamientos de vidrio
}

\author{
E. ESCUDER SILLA, J. ALBA FERNÁNDEZ, J. RAMIS SORIANO \\ Escuela Politécnica Superior de Gandia, Universidad Politécnica de Valencia, \\ Crtra. Nazaret-Oliva (S / N), 46730 Gandia \\ España
}

\begin{abstract}
En este artículo se aplica el modelo de Ookura \& Saito para determinar el aislamiento acústico a ruido aéreo de sistemas constructivos basados en vidrios. En concreto, los cálculos que se presentan son para vidrios monolíticos de distintos espesores y para vidrios laminados de diferentes tipos. Existen diferentes modelos de predicción del comportamiento acústico a ruido aéreo de estructuras multicapa (y los vidrios laminados pueden considerarse como tales). En todos ellos, los datos de entrada son las constantes elásticas y el factor de pérdidas. Tanto los vidrios monolíticos como la capa intermedia se han caracterizado siguiendo diferentes normativas. Los resultados se comparan con medidas experimentales y con datos recogidos del estudio de Marsh (1), obteniéndose un grado de ajuste aceptable
\end{abstract}

Palabras clave: acristalamientos de vidrio monolítico y laminado, aislamiento acústico, prediccion.

\section{Airborne Sound Insulation of Glazing Systems.}

In this paper, the Ookura \& Saito model is applied to determine the Airborne Sound Insulation of glazing systems. In particular, the calculations that appear are for monolithic glasses of different thicknesses and laminated glasses from different types. There are different prediction models of the airborne acoustic behaviour of multilayer panels (and the laminated glasses can be considered like such). In all of them, the input data are the elastic constants and the loss factor. The monolithic glasses and the intermediate layer have been characterized according to different Standards. The results are compared with experimental measurements and data of the study of Marsh (1), obtaining a range of acceptable adjustment.

Keywords: glazing systems, Airborne Sound Insulation, prediction.

\section{INTRODUCCIÓN}

La sociedad demanda mayores garantías de confort de las viviendas. Dentro de estas demandas de confort se puede incluir la necesidad de un nivel suficiente de aislamiento acústico, con el objeto de evitar bien la intromisión de sonidos no deseados provenientes del exterior o la salida de sonido al exterior. En definitiva, el objetivo es aumentar al máximo el índice de aislamiento de una cierta configuración, intentando disminuir en lo posible los costes. Por otra parte, es cada vez más frecuente la utilización de sistemas constructivos basados en vidrios.

El objeto de este trabajo es el estudio del aislamiento acústico a ruido aéreo de estructuras multicapa de vidrios. La validación de las teorías sobre el aislamiento acústico lleva consigo el ensayo de diferentes materiales y configuraciones. El estudio experimental del comportamiento acústico de distintas configuraciones suele realizarse en cámara de transmisión, cuya descripción se puede encontrar en la norma UNE-EN ISO 140-1, "Medición del aislamiento acústico en los edificios y de los elementos de construcción. Parte 1: Requisitos de las instalaciones del laboratorio sin transmisiones indirectas", (2). El procedimiento para realizar las medidas se describe en la norma UNE-EN ISO 140-3,
"Medición del aislamiento acústico en los edificios y de los elementos de construcción. Parte 3: Medición en laboratorio del aislamiento acústico al ruido aéreo de los elementos de construcción", (3). Entre distintos laboratorios las diferencias en la medida del aislamiento acústico a ruido aéreo de un mismo acristalamiento pueden alcanzar hasta $3 \mathrm{~dB}$ según la norma UNE-EN 20140-2, "Medición del aislamiento acústico en los edificios y de los elementos de edificación. Parte 2: Determinación, verificación y aplicación de los datos de precisión" (4). Esta norma proporciona una guía para determinar los valores de repetibilidad y reproducibilidad, y verificar esos valores para distintas configuraciones de medida en un mismo laboratorio y para comparaciones entre distintos laboratorios. Por esta razón y por motivos básicos relacionados con una planificación, es conveniente disponer de herramientas de predicción que nos acerquen con la menor desviación posible a las medidas reales. Además, a veces no es necesario obtener resultados absolutos sino que basta con saber si una solución constructiva aísla más que otra.

Diversas teorías describen el comportamiento acústico de estructuras múltiples. Entre éstas destacan el modelo de Ookura \& Saito (5), que se basa en el acoplo de impedancias 
entre distintas capas, el desarrollado por Trochidis \& Kalaroutis (6) o Bruneau (7), basadas en la transformada de Fourier espacial, y otros trabajos como el de Lauriks basados en la teoría de Biot (8), el de Croker \& Price sobre el modelo SEAStatistical Energy Análisis- (9) o los basados en la técnica de los elementos finitos de Panneton \& Atalla (10). En el trabajo de Trochidis \& Kalaroutis se desarrolla un método con el que se obtiene una matriz que define la configuración multicapa (6). Está basado en la aplicación de las condiciones de contorno entre capas, obteniendo distintas ecuaciones en derivadas parciales, que pueden transformarse en ecuaciones algebraicas mediante la Transformada de Fourier Espacial. Una vez en formato algebraico, estas ecuaciones se resuelven permitiendo obtener información sobre todas las variables acústicas de la configuración multicapa.

En acristalamientos de vidrio nos encontramos con soluciones cuyas capas pueden corresponder a los materiales siguientes:

a) Vidrios monolíticos, cuyas características generales vienen descritas en las normas UNE-EN 1748-1, "Vidrio para la edificación. Productos básicos especiales. Parte 1: Vidrios borosilicatados.",UNE-EN 12758, " Vidrio para la construcción. Acristalamiento y aislamiento al ruido aéreo. Definiciones y determinación de las propiedades", y UNE-EN 572-1, "Vidrio para la construcción. Productos básicos de vidrio. Vidrio de silicato sodocálcico. Parte 1: Definiciones y propiedades generales físicas y mecánicas", (11-13) y que, desde el punto de vista acústico se comportan como capas impermeables, en las que la onda acústica provoca una vibración en la placa y la energía reflejada es grande, estudiadas por Ookura, Trochidis y Alba $(5,6,14)$.

b) Lámina amortiguante derivada de un compuesto orgánico, tipo PVB (Polibutil-Vinilo -Butiral), o de resinas de metacrilato, PMMA (PoliMetil-MetAcrilato)), cuya misión es atenuar la transmisión de la vibración. Los parámetros relevantes son el factor de pérdidas y las características elásticas.

c) Aire o gas, caracterizado por su densidad, velocidad de propagación del sonido, y factor de atenuación con la distancia. No es objeto del presente estudio. Estas cámaras de aire actúan como un resorte elástico dentro del conjunto de la estructura, influyendo en sus propiedades generales.

En el estudio que nos ocupa, como se ha comentado más arriba, nos centraremos en vidrios monolíticos y en vidrios laminados (combinaciones de monolíticos y láminas PVB).

En este trabajo se utiliza el modelo de Ookura \& Saito para obtener el aislamiento acústico de acristalamientos de vidrios, y se evalua su grado de ajuste con medidas de aislamiento acústico realizadas en cámara de transmisión y obtenidas de las referencias. Se consideran como parámetros de entrada del software el factor de pérdidas, la frecuencia de resonancia y el módulo de Young medidos en laboratorio de cada uno de los vidrios, estos datos generalmente se consideran teóricos. Se ha elegido este modelo desde el punto de vista matemático por su potencia y sencillez, y porque tiene en cuenta además de las impedancias, los parámetros del material absorbente como son las constantes de propagación. Otros modelos como el del acoplo de impedancias de Bruneau se basan únicamente en las impedancias y no consideran estas constantes de propagación necesarias para caracterizar el material. El modelo de Trochidis \& Kalaroutis si que considera las constantes de propagación, pero matemáticamente es más complejo ya que tiene que resolver matrices.

\section{FUNDAMENTOS}

\subsection{Aislamiento acústico a ruido aéreo y su medida}

Los mecanismos empleados en el aislamiento acústico serán los que dificulten la propagación del sonido, o en términos energéticos, los que consigan atenuar la energía de las ondas acústicas. Para el control de la transmisión del sonido a un local objeto del aislamiento acústico hay que tener en cuenta que el mecanismo de transmisión está formado por tres elementos básicos, que son: la fuente de ruido, el canal de transmisión (ventanas, paredes, etc..) y el local receptor como comenta Llinares (15).

El parámetro a, que mide el aislamiento acústico de un material o dispositivo se denomina aislamiento acústico específico (medible únicamente en laboratorio), y viene dado por la ecuación:

$$
a=10 \log \left(\frac{I_{i}}{I_{t}}\right)
$$

donde $I_{i}$ es la intensidad acústica incidente e $I$, es la intensidad acústica transmitida. La relación entre estas intensidades proporciona el coeficiente de transmisión del cerramiento, $\boldsymbol{\tau}$, según la ecuación,

$$
\tau=\frac{I_{i}}{I_{t}}
$$

que dependerá del ángulo de incidencia de la onda sonora y de la frecuencia. Las pérdidas por transmisión que presenta una determinada capa, denominadas con el acrónimo TL (Transmisión Loss), se obtienen directamente de la ecuación

$$
T L=10 \cdot \log (1 / \tau)
$$

una vez conocido el coeficiente de transmisión. Debido a la difusión que se produce en un recinto, es importante considerar que por encima de un cierto ángulo el sonido no se recibe, por lo que resultará necesario ponderar este coeficiente hasta un cierto ángulo límite.

El procedimiento de medida del aislamiento acústico a ruido aéreo de las pérdidas por transmisión de particiones viene descrito en la norma UNE-EN ISO 140-4, "Medición del aislamiento acústico en los edificios y de los elementos de construcción.. Parte 4: Medición "in situ" del aislamiento al ruido aéreo entre locales" (16). En la figura 1 se muestra una posible configuración. A grandes rasgos, el procedimiento consiste en generar un ruido en el recinto emisor según se indica en la normativa, buscando crear un campo sonoro lo más homogéneo posible.

La medida promediada del nivel de presión sonora en el recinto emisor, $\mathrm{L}_{1}$, menos la medida promediada (y corregida, en su caso, respecto al nivel de fondo) del nivel de presión sonora en el recinto recepctor, $\mathrm{L}_{2}$, es lo que se conoce como aislamiento acústico bruto, D:

$$
D=L_{1}-L_{2}
$$




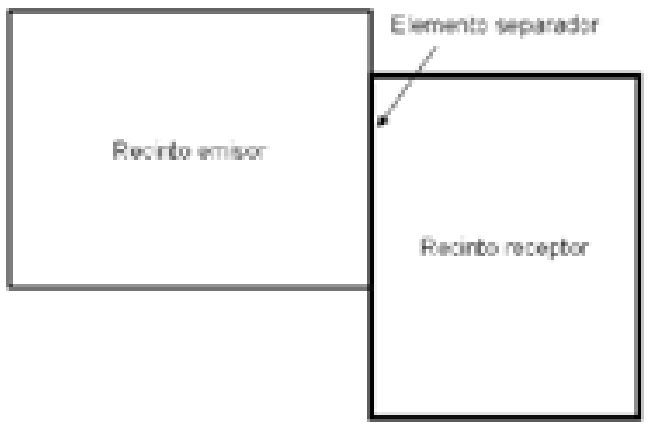

Fig. 1- Ejemplo de partición a medir.

\subsection{Parámetros relevantes en el comportamiento acústico de vidrios.}

En el modelo que se utiliza en este trabajo, los parámetros necesarios para determinar el amortiguamiento que se produce en un vidrio son el factor de pérdidas y los módulos elásticos. Para comprender mejor estos conceptos resulta conveniente comentar, en primer lugar, algunos aspectos relacionados con los sistemas amortiguados. En efecto, el transitorio de caída de la amplitud de la oscilación para el amortiguamiento en los sistemas de un grado de libertad tiene la forma que se presenta en la figura. 2. Uno de los parámetros que se suelen utilizar para describir este amortiguamiento es el tiempo de relajación, $\xi$, es el tiempo necesario para que la amplitud disminuya 1 /e desde su valor inicial.

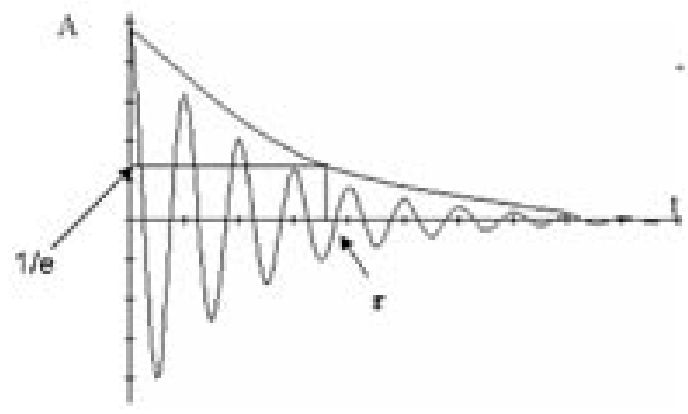

Fig. 2- Amplitud de la oscilación de una señal no forzada. Tiempo de relajación.

Karjalainen en uno de sus trabajos estudia el tiempo de reverberación mecánico $\left(\mathrm{T}_{60}\right)$ que se define como el lapso de tiempo que debe transcurrir, una vez que ha cesado la excitación mecánica, para que el desplazamiento $x(t)$ de la masa del sistema vibratorio, disminuya a la millonésima parte de su valor inicial (17). Se denomina Lx al nivel de desplazamiento que se expresa según la ecuación

$$
L x=10 \log \left(\frac{x(t)}{x_{r / f}}\right)
$$

mediante la relación logarítmica entre el desplazamiento en un determinado tiempo y el desplazamiento de referencia. En términos de nivel de desplazamiento $L \boldsymbol{x}, \mathrm{T}_{60}$ corresponde al tiempo que tarda el nivel de desplazamiento en decaer $60 \mathrm{~dB}$. En general, no se dispone de un rango dinámico tan grande, por lo que el tiempo de reverberación se determina extrapolando el valor calculado para la caída de 20 ó $30 \mathrm{~dB}$.

Resulta interesante poder relacionar el factor de pérdidas y el tiempo de reverberación mediante la ecuación:

$$
T_{60}=\frac{2,2}{\eta \cdot f_{n}}
$$

donde $\mathrm{f}_{\mathrm{n}}$ es la frecuencia de resonancia del sistema y $\eta$ el factor de pérdidas.

El factor de pérdidas, $\boldsymbol{\eta}$, se define como la razón entre la energía disipada por radian y la energía potencial máxima que el sistema puede almacenar en un ciclo de vibración según la ecuación:

$$
\eta=\frac{D}{2 \pi W}
$$

$W$ es la energía total del sistema, D corresponde a la energía disipada por ciclo de vibración a la frecuencia $\omega_{n}$ y de amplitud X. El factor de pérdidas, $\eta$, se puede calcular a partir del ancho de banda de potencia mitad según la expresión suiguiente y a partir de la figura 3:

$$
\eta=\frac{f_{2}-f_{1}}{f_{0}}
$$

donde $f_{0}$ es la frecuencia natural del pico y $f_{2}$ y $f_{1}$ son las frecuencias superiores e inferiores límite de caída $3 \mathrm{~dB}$, respectivamente.

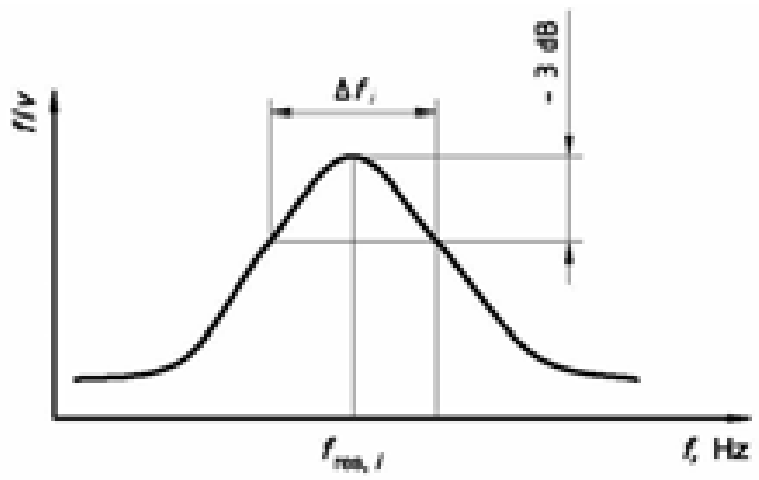

Fig. 3- Caída de ancho de banda 3dB [19].

Cuando un cristal se excita, la frecuencia a la cual la velocidad de propagación de la onda de flexión libre en el panel se iguala a la velocidad de la onda acústica en el aire se define como frecuencia crítica según Renji (18). La respuesta de vibración de un panel es mayor alrededor de la frecuencia crítica. Por tanto para obtener la respuesta de una estructura a una excitación acústica es necesario conocer exactamente esta frecuencia crítica. Esto se utiliza en diseño, ya que una estructura puede diseñarse para que su frecuencia crítica esté fuera del rango de frecuencias en el que la excitación 
acústica es mayor. El factor de pérdidas, es máximo justo a la frecuencia crítica.

El módulo de Young de un vidrio, E, gobierna los fenómenos de flexo-tracción, y el valor de G (módulo de cizalladura o de elasticidad transversal) contempla el comportamiento de los posibles deslizamientos entre-capas, y el coeficiente ' $v$ ' de Poisson la contracción lateral. Estos tres parámetros están íntimamente relacionados a través de la ecuación

$$
G=\frac{E}{2(1+v)}
$$

Conocido el valor del módulo de Young de un determinado vidrio es posible determinar la frecuencia crítica a partir de la ecuación

$$
f_{c}=\frac{c^{2}}{2 \pi} \sqrt{\frac{m}{D}}
$$

donde $\mathrm{D}$ es la rigidez a la flexión del material, $\mathrm{f}_{\mathrm{c}}$ la frecuencia crítica, c la velocidad del sonido y m es la masa por unidad de superficie del vidrio.

\section{3- Procedimientos para caracterización de vidrios monolíticos y laminados}

Fundamentalmente se utilizan las tres normativas siguientes:

La norma ISO/PAS 16940:2004 (E), “Glass in building - Glazing and airborne sound insulation - Measurement of the mechanical impedance of laminated glass", describe un método de medida del factor de pérdidas y el módulo de rigidez a flexión equivalente de muestras de vidrio laminado (19). Además proporciona una ecuación para obtener las pérdidas por transmisión en este tipo de particiones.

La norma ASTM/C 623-92, "Standard Test Method for Young's Modulus, Shear Modulus, and Poisson's Ratio for Glass-Ceramics by Resonance", que establece un método dinámico para la determinación del módulo de Young, módulo de cizalladura y el coeficiente de Poisson (20). A partir de los datos geométricos, la densidad y las frecuencias de resonancia de una muestra se obtienen las propiedades elásticas de ese material. El módulo de Young se determina a partir de las frecuencias de resonancia en el modo de vibración a flexión. Todos los materiales formados por vidrio y vidrio-cerámico que sean elásticos, homogéneos e isótropos pueden analizarse mediante este método.

La normativa ASTM/E 756-98, "Standard Test Method for Measuring Vibration-Damping Properties of Materials", permite determinar tanto el módulo de Young como el de cizalladura de materiales del tipo elastómero, cuando está confinado entre dos láminas rígidas que se deforman a flexión conocido el factor de pérdidas (21).

En las tres normas citadas, el dispositivo experimental de medida permite apoyar la viga del cristal a estudiar bien por su centro, bien en dos puntos tal como indica en la figura 4 , o bien por uno de sus extremos. La muestra es excitada mecánica o acústicamente (excitador o altavoz en su caso), y se registra la señal correspondiente a la respuesta vibratoria del sistema, mediante un acelerómetro o mediante cualquier otro tipo de sensor situado en el extremo libre de la barra.

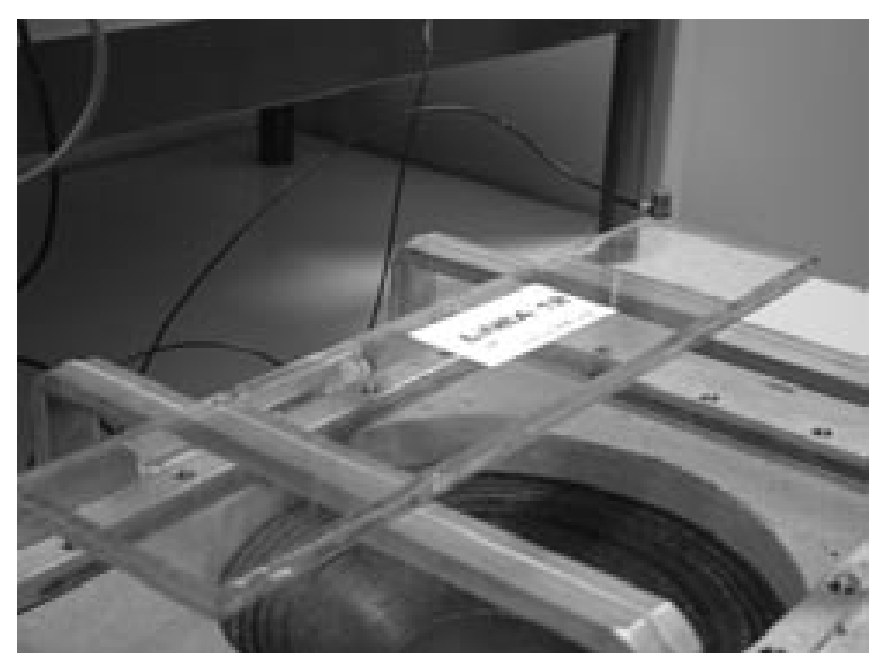

Fig. 4- Dispositivo experimental de medida según la norma C 623-92.

\subsection{Predicción según el modelo de Ookura\&Saito}

El modelo de Ookura \& Saito predice el aislamiento acústico de un número determinado de particiones multicapa. Interesa conocer la relación entre la presión acústica incidente sobre la primera capa y la presión acústica transmitida a través de todas las capas. El modelo se basa en obtener las relaciones entre las presiones acústicas a través de las impedancias características asociadas a cada capa y que se pueden calcular fácilmente. Con este modelo se obtienen las expresiones del índice de debilitamiento para paredes múltiples construidas con varias combinaciones de capas impermeables, cámaras de aire y materiales absorbentes.

El modelo de predicción del comportamiento acústico de estructuras multicapa considera una estructura general de paredes múltiples infinitas, tal y como se observa en la figura 5. Dicha estructura constituida por $\mathrm{N}$ elementos y cada uno de los cuales puede ser una capa impermeable, una cámara de aire o un material absorbente. Se denomina $\mathrm{p}_{\mathrm{i}}$ a la onda

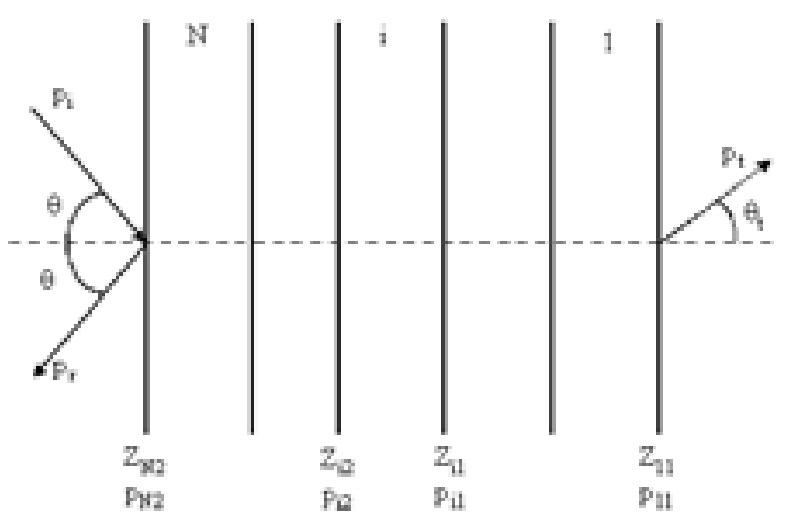

Fig. 5- Modelo multicapa.

de presión incidente y $\mathrm{p}_{\mathrm{r}}$ a la onda de presión reflejada. Se supone que una onda plana incide sobre la cara izquierda del elemento $\mathrm{N}$-ésimo con un ángulo de incidencia $\theta$. La onda 
que incide por la parte izquierda continuará propagándose a través de la estructura y radiará por la cara derecha del primer elemento como una onda plana de presión $\mathrm{p}_{\mathrm{t}}$ hacia un campo libre con un ángulo de transmisión $\theta_{t}$. En el análisis se numera con el subíndice $\mathrm{i}=1,2, \ldots, \mathrm{n}$ cada uno de los parámetros físicos del i-ésimo elemento, y se utiliza un segundo subíndice para indicar la cara considerada, 2 para la cara izquierda del elemento y 1 para la cara derecha.

El cociente entre la presión en la superficie de incidencia $\mathrm{p}_{\mathrm{N} 2}$ y la presión incidente $\mathrm{p}_{\mathrm{i}}$ es la siguiente:

$$
\frac{p_{N 2}}{p_{i}}=\frac{p_{i}+p_{r}}{p_{i}}=\frac{2 Z_{N 2}}{Z_{N 2}+\frac{p c}{\cos \theta}}
$$

donde $\mathrm{Z}_{\mathrm{N} 2}$ es la impedancia acústica normal vista desde la cara izquierda de la superficie del n-ésimo elemento y

$$
\frac{\rho c}{\cos \theta} \text { es la impedancia acústica normal en campo libre }
$$

de una superficie con incidencia oblicua, la cual es igual a la relación de impedancias del primer elemento $Z_{11} \rho$ es la densidad del aire y c es la velocidad del sonido. Partiendo de las condiciones de presión en cada superficie, la expresión del factor de transmisión para incidencia oblicua resulta:

$$
\tau(\theta)=\left|\frac{p_{t}}{p_{i}}\right|^{2}-\left|\frac{p_{11}}{p_{i}}\right|^{2}-\left|\frac{p_{N 2}}{p_{i}}\right|^{2}\left|\frac{p_{N 1}}{p_{N 2}} \cdots \frac{p_{11}}{p_{i 2}} \cdots \frac{p_{11}}{p_{12}}\right|^{2}
$$

Las relaciones de presiones se pueden obtener a partir de las características de impedancia de cada superficie. De este modo, se obtienen las pérdidas por transmisión según la ecuación:

$$
T L=10 \log \frac{1}{\tau(\theta)}
$$

Para obtener las pérdidas por transmisión para incidencia aleatoria, también llamado índice de debilitamiento, es necesario promediar para todos los factores de transmisión y ángulos de incidencia, de acuerdo con la expresión:

$$
R_{d}=10 \log \left[\frac{\int_{0}^{\theta_{\gamma}} \cos \theta \operatorname{sen} \theta \cdot d \theta}{\int_{0}^{\theta^{\prime}} \tau(\theta) \cos \theta \operatorname{sen} \theta \cdot d \theta}\right]
$$

donde $\theta_{1}$ es el ángulo límite por encima del cual se supone que el sonido no se recibe. Dicho ángulo varía entre $70^{\circ}$ y $85^{\circ}$. En concreto, en la norma ISO/PAS 16940:2004 se especifica que para cristales el ángulo límite es de $75^{\circ}$.

En el caso que nos ocupa se tratan dos tipos de láminas impermeables, vidrios y láminas amortiguantes (PVB o PMMA). La diferencia más notable existente entre ellas radica en el valor del factor de pérdidas, que resulta mayor para las láminas amortiguantes que para los vidrios.

Se considera que las vibraciones de una placa infinita de espesor $h$ producen una diferencia de presiones a ambos lados de la placa. En este caso, la velocidad de la placa en la dirección x se puede escribir como:

$$
u=\frac{p_{2}-p_{1}}{Z_{\text {su }}}
$$

donde $p_{2}$ es la presión sonora que incide sobre la superficie en $\mathrm{x}=0, \mathrm{p}_{1}$ es la presión que se transmite en $\mathrm{x}=\mathrm{h} \mathrm{y} \mathrm{Z}_{\mathrm{m}}$ es la llamada impedancia de superficie de la placa y viene dada por:

$$
Z_{m}=\eta \operatorname{con}\left(\frac{\omega}{\omega_{c}}\right)^{2} \operatorname{sen}^{4} \theta+j \omega m\left[1-\left(\frac{\omega}{\omega_{c}}\right)^{2} \operatorname{sen}^{4} \theta\right]
$$

donde $\eta$ es el factor de pérdidas de la placa, $\omega$ es la frecuencia angular de la onda sonora, m es la masa por unidad de área y $\omega_{c}$ es la frecuencia angular crítica de la placa. En la superficie de la placa la velocidad a ambos lados debe ser la misma que la velocidad de la propia placa. Teniendo en cuenta todo esto se pueden obtener las siguientes expresiones:

$$
\begin{aligned}
& Z_{2}=Z_{1}+Z_{\text {m }} \\
& \frac{p_{2}}{p_{1}}=\frac{Z_{2}}{Z_{1}}
\end{aligned}
$$

donde $Z_{2}$ es la impedancia acústica normal a la superficie en $\mathrm{x}=0, \mathrm{y} Z_{1}$ es la impedancia acústica normal en $\mathrm{x}=\mathrm{h}$.

\section{DESARROLLO}

Con el objeto de evaluar las pérdidas por transmisión de acristalamientos se ha desarrollado un programa de predicción del aislamiento acústico basado en el modelo de Ookura\&Saito en Matlab. En la figura 6, se presenta el diagrama de flujo que evalúa las pérdidas por transmisión de una capa impermeable usando el modelo de Ookura\&Saito. Los datos de entrada del vidrio se conocen, donde $\mathrm{h}$ es el espesor, $\mathrm{m}$ es la masa por unidad de área, D es la rigidez a flexión y $\eta$ es el factor de pérdidas, $\mathrm{lim}$ representa el ángulo límite en grados, f la frecuencia y $N$ los pasos de integración angular. Calculando la relación de impedancias y realizando la integración discreta,

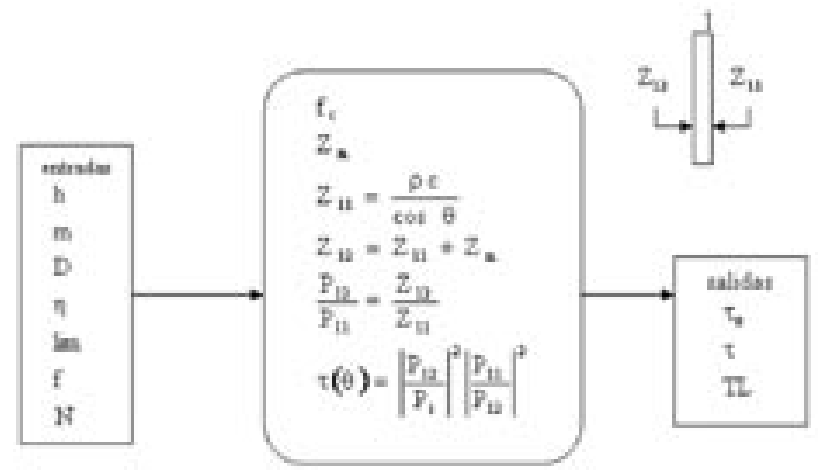

Fig. 6- Diagrama de flujo del programa que evalúa las pérdidas por transmisión y el coeficiente de transmisión de un vidrio con el modelo de Ookura \& Saito. 
se obtienen como parámetros de salida $\boldsymbol{\tau}_{\theta}$ (Coeficiente de transmisión (en función del ángulo)) y $\boldsymbol{\tau}$ (Coeficiente de transmisión (campo difuso)), así como las pérdidas por transmisión del vidrio. Para realizar la integral discreta de aislamiento se ha utilizado el método de los rectángulos por su rapidez de cálculo. Este método calcula el área que existe bajo la curva realizando una aproximación de esta área como la suma de un número finito de rectángulos. El proceso de sumar es muy rápido pues no existe ningún bucle anidado. Se puede conseguir suficiente exactitud aumentando el número de intervalos en los que se divide el intervalo de integración. En la figura 7, se presenta el diagrama de flujo que evalúa las pérdidas por transmisión de un vidrio laminar con el modelo de Ookura \& Saito. Los datos de entrada de los vidrios son similares al caso de un vidrio simple, con la diferencia de que en este caso se consideran los datos del absorbente. Se utilizan el grosor del aborbente, $d$, y los parámetros que caracterizan un material absorbente: $k_{b}$, número de onda complejo, y $\rho_{b}$, densidad compleja del absorbente, que se pueden calcular con los modelos de Miki, Delany \& Bazley o Allard \& Champoux (22-24). Del mismo modo que en el algoritmo anterior, se calcula la integral discreta y se obtienen las pérdidas por transmisión del vidrio laminar. Para construir la base de datos de entrada del software de los parámetros elásticos de los vidrios monolíticos y laminados considerados se han utilizado

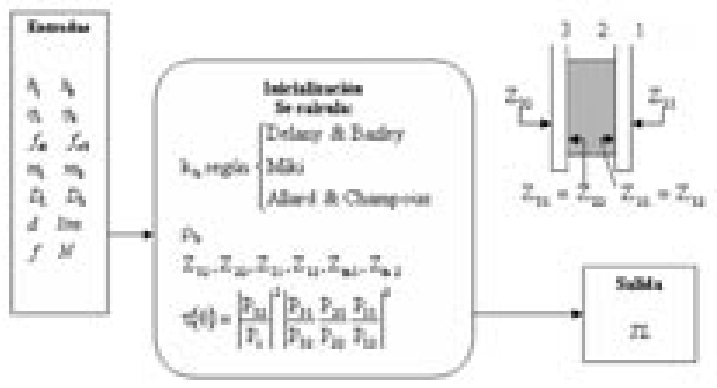

Fig. 7- Diagrama de flujo del programa que evalúa las pérdidas por transmisión de un vidrio laminar con el modelo de Ookura \& Saito.

dos fuentes: por una parte se ha realizado una campaña de medidas según las normativas comentadas en el punto 2.3, y por otra una búsqueda bibliográfica. Algunos de los resultados obtenidos de frecuencia de resonancia, factor de pérdidas y módulo de Young de los vidrios monolíticos se presentan en las tablas I y II. En la mayoría de referencias consultadas sobre vidrios laminados, Dharani, Wei, Duser, Behr, Du Bois, Vallabhan y Asik, las características elásticas que presentan los compuestos de PVB utilizados son las siguientes: el módulo de Young (E) oscila entre 0.5 y $7 \mathrm{MPa}$, el módulo de cizalladura (G) presenta valores entre 1 y $20 \mathrm{MPa}$ para una temperatura de entre $10^{\circ}$ y $30^{\circ} \mathrm{C}$. No hay que olvidar que el módulo de cizalladura depende fuertemente de la temperatura (25-33). Considerando estos datos de entrada se han obtenido las predicciones de las pérdidas por transmisión de diferentes configuraciones estudiadas.

En las figuras 8 y 9 se presentan las pérdidas por transmisión de los resultados de predicción obtenidos con el software para un vidrio simple y uno doble. Se observa, en líneas generales, una diferencia de unos $5 \mathrm{~dB}$ de un vidrio simple a uno doble en la mayoria de frecuencias. Se aprecia que para el vidrio de $10 \mathrm{~mm}$ el mínimo aislamiento está sobre $800 \mathrm{~Hz}$ y para el vidrio doble se desplaza a $1 \mathrm{kHz}$. El índice de reducción sonora global del vidrio simple es de $30 \mathrm{~dB}$ y del vidrio doble de $35 \mathrm{~dB}$, observándose una diferencia de $5 \mathrm{~dB}$ en los valores globales.

TABLA I. FRECUENCIA DE RESONANCIA FUNDAMENTAL Y FACTOR DE PÉRDIDAS DE UNA BARRA DE VIDRIO MONOLÍTICO EN FUNCIÓN DE SU ESPESOR. NORMA ISO/PAS 16940

\begin{tabular}{|l|c|c|c|c|}
\hline $\mathrm{h}(\mathrm{mm})$ & 3 & 5 & 8 & 10 \\
\hline$f_{0}(\mathrm{~Hz})$ & 229 & 309,5 & 489 & 608 \\
\hline$\eta$ & 0,094 & 0,052 & 0,032 & 0,008 \\
\hline
\end{tabular}

TABLA II. MÓDULO DE YOUNG PARAVIDRIOS MONOLÍTICOS EN FUNCIÓN DE DIFERENTES ESPESORES SEGÚN LA NORMA C 623-92.

\begin{tabular}{|l|c|c|c|c|}
\hline $\mathrm{h}(\mathrm{mm})$ & 3 & 5 & 8 & 10 \\
\hline $\mathrm{E}(\mathrm{N} / \mathrm{m} 2)$ & $6,93 \mathrm{E}+10$ & $6,74 \mathrm{E}+10$ & $6,77 \mathrm{E}+10$ & $6,65 \mathrm{E}+10$ \\
\hline
\end{tabular}

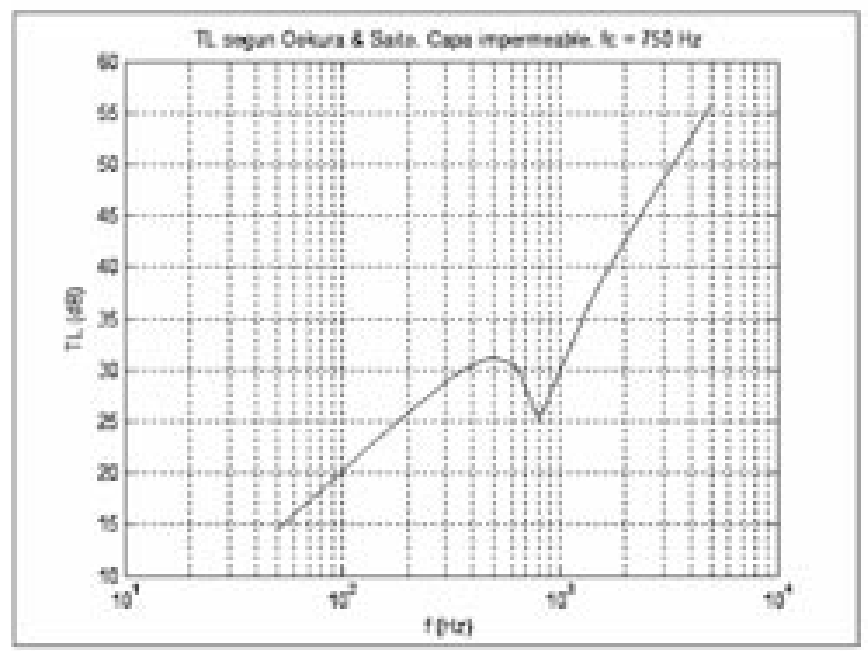

Fig. 8- Perdidas por transmisión obtenidas con el software para un vidrio de $10 \mathrm{~mm}$.

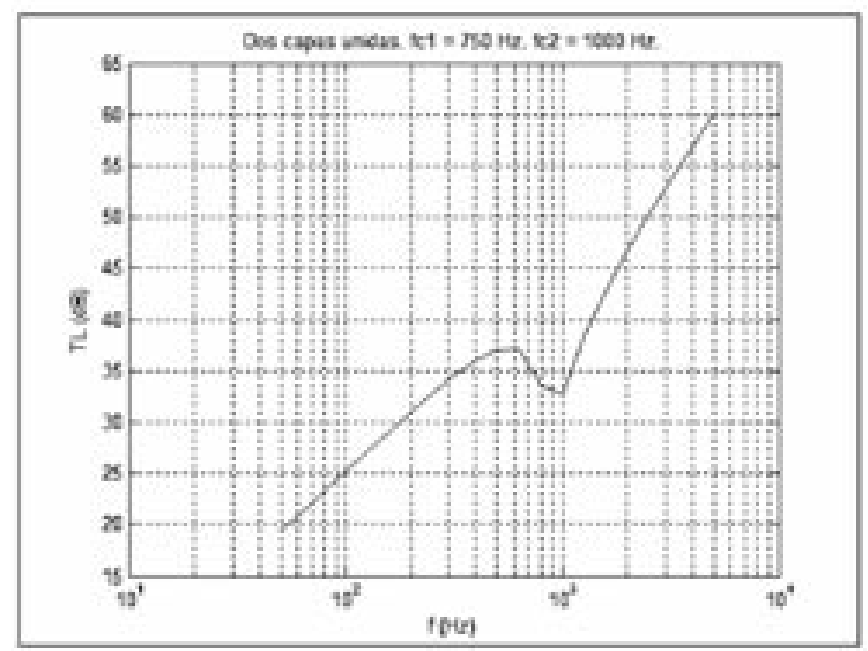

Fig. 9- Perdidas por transmisión obtenidas con el software para un vidrio de $8 \mathrm{~mm}$ y un vidrio de $10 \mathrm{~mm}$ unidos. 
El método convencional de cálculo del aislamiento acústico se denomina "ley de masas", ésta sólo depende de la masa por unidad de superficie del elemento a considerar, para masas $m<150 \mathrm{~kg} / \mathrm{m}^{2}$ el índice de reducción sonora global en $\mathrm{dB}(\mathrm{A})$ se calcula según

$$
R=16.6 \log m+2
$$

Para el caso del vidrio simple de $10 \mathrm{~mm}$, la masa del vidrio es de $25 \mathrm{~kg} / \mathrm{m}^{2}$ y el índice de reducción sonora global obtenido con el modelo de Ookura \& Saito resulta ser de 28.5 $\mathrm{dB}(\mathrm{A})$ o $30 \mathrm{~dB}$, sin embargo utilizando la ley de masas se obtiene un índice de reducción sonora global de $25 \mathrm{~dB}(\mathrm{~A})$, que comparando con valores medidos en laboratorio (29 $\mathrm{dB}(\mathrm{A})$ ) evidencia la necesidad de utilizar modelos más precisos que consideren las características elásticas de cada material utilizado frente a la ley de masas convencional. Además la ley de masas da un único resultado global y no muestra la información por frecuencias que resulta de gran utilidad para solucionar un problema acústico.

En las figuras 10 y 11, se presenta una comparación de las pérdidas por transmisión de los resultados de predicción obtenidos con el programa realizado, con medidas experimentales y con datos obtenidos de la bibliografía, para los casos de un vidrio monolítico de $12 \mathrm{~mm}$ de espesor y de un vidrio laminado de $3 \mathrm{~mm}$ y un PVB de $0.38 \mathrm{~mm}$, respectivamente. En la tabla III se presentan los valores globales de aislamiento acústico para el caso de la configuración multicapa vidrio $3 \mathrm{~mm}+$ PVB + vidrio $3 \mathrm{~mm}$ presentados en la figura 11. Se observa que el grado de ajuste es aceptable. En las figuras 12 y 13 se presentan las barras de error de las pérdidas por transmisión del software respecto a las medidas experimentales y respecto a los valores de la referencia (1) para el caso de un vidrio laminado de $3 \mathrm{~mm}$ y un PVB de 0.38 $\mathrm{mm}$, respectivamente. El error de las pérdidas por transmisión representado en las barras de error para la comparativa entre las medidas experimentales y el software es de $\pm 1.5 \mathrm{~dB}$ para el rango de frecuencias de160 Hz a $1.6 \mathrm{kHz}$, aumenta hasta $\pm 2.5 \mathrm{~dB}$ de $2 \mathrm{kHz}$ a $4 \mathrm{kHz}$ y alcanza valores de hasta $\pm 5 \mathrm{~dB}$ para frecuencias menores de $160 \mathrm{~Hz}$. En la comparativa entre la referencia (1) y el software, el error, en general, es menor, de $\pm 0.5 \mathrm{~dB}$ para el rango de frecuencias de $250 \mathrm{~Hz}$ a $1.6 \mathrm{kHz}$, aumenta hasta $\pm 2 \mathrm{~dB}$ de $2 \mathrm{kHz}$ a $4 \mathrm{kHz}$ y alcanza valores de

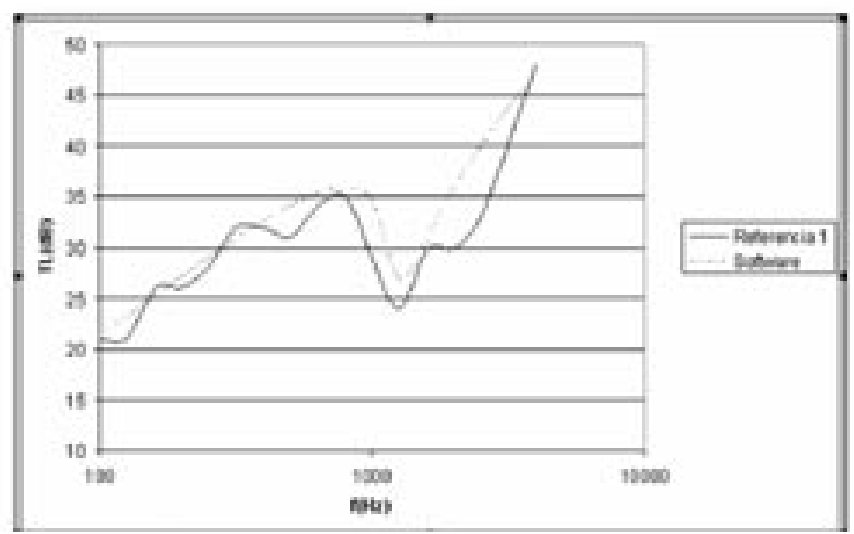

Fig. 10- Comparación de las pérdidas por transmisión de un vidrio monolítico de $12 \mathrm{~mm}$ entre la predicción obtenida con el programa desarrollado en Matlab (Software) y la referencia obtenida de la bibliografia para dos vidrios de espesores cercanos (1).

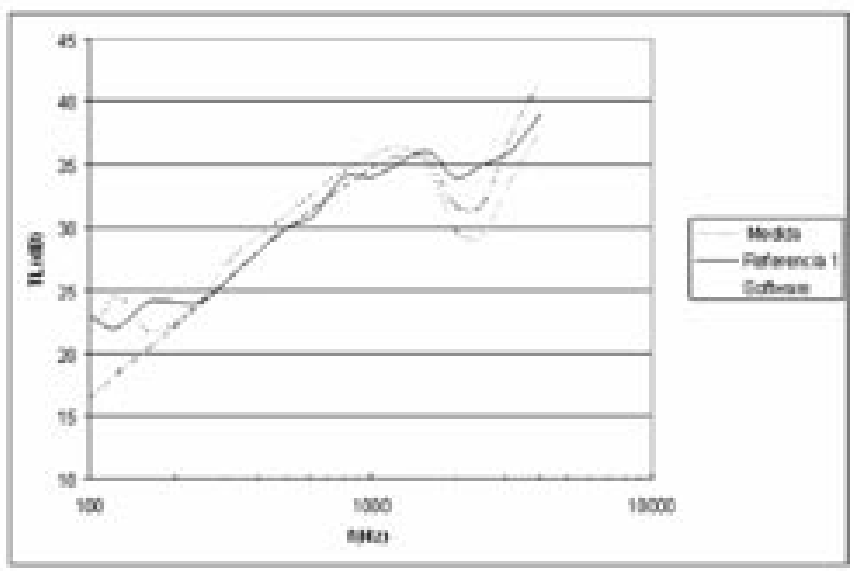

Fig. 11- Comparación de las pérdidas por transmisión de la configuración $3 \mathrm{~mm}+\mathrm{PVB}+3 \mathrm{~mm}$ entre la predicción obtenida con el programa desarrollado en Matlab (Software), las medidas realizadas en cámara de transmisión (Medida) y la referencia obtenida de la bibliografia para el vidrio laminado $3.2 \mathrm{~mm}+\mathrm{PVB}+3.2 \mathrm{~mm}$ (1).

TABLA III. ÍNDICE DE REDUCCIÓN SONORA GLOBAL OBTENIDO CON DIFERENTES MÉTODOS.

\begin{tabular}{|l|c|}
\hline & Vidrio $3 \mathrm{~mm}+$ PVB + Vidrio $3 \mathrm{~mm}$ \\
\hline Software predicción & 33.0 \\
\hline Referencia bibliográfica & 33.5 \\
\hline Medida experimental & 33.0 \\
\hline
\end{tabular}

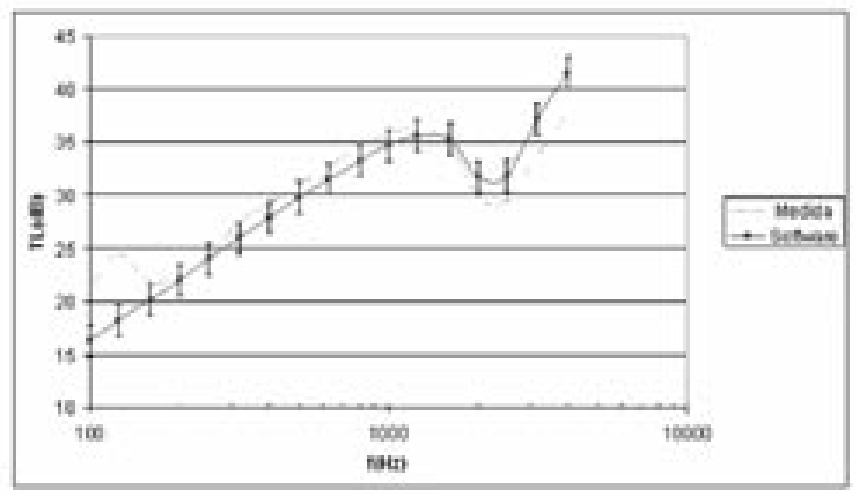

Fig. 12- Barras de error de las pérdidas por transmisión de $\pm 1.5 \mathrm{~dB}$ de la predicción obtenida con el programa desarrollado en Matlab (Software) respecto a las medidas realizadas en cámara de transmisión (Medida) para la configuración 3mm+PVB+3mm.

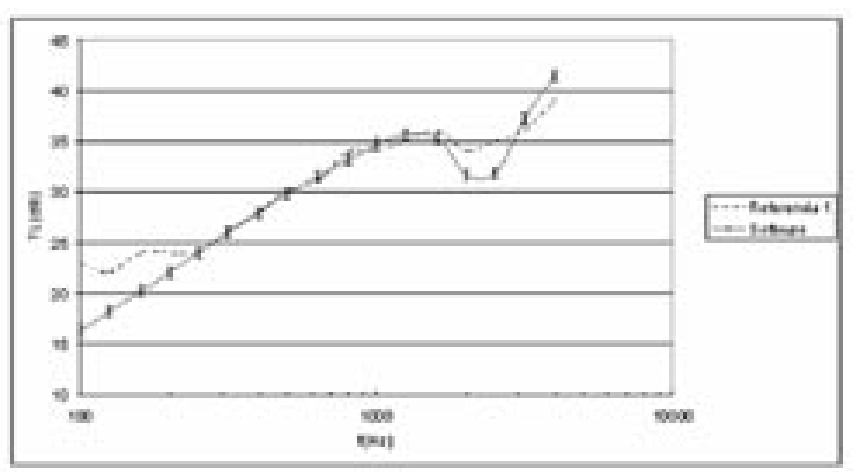

Fig. 13- Barras de error de las pérdidas por transmisión de $\pm 0.5 \mathrm{~dB}$ de la predicción obtenida con el programa desarrollado en Matlab (Software) respecto a la referencia obtenida de la bibliografia para el vidrio laminado $3.2 \mathrm{~mm}+\mathrm{PVB}+3.2 \mathrm{~mm}(1)$. 
$\pm 4 \mathrm{~dB}$ para frecuencias menores de $250 \mathrm{~Hz}$. Se observa que el error es mayor a bajas y altas frecuencias, y en el rango de frecuencias medio no sobrepasa los $\pm 3 \mathrm{~dB}$ lo que puede considerarse admisible.

\section{CONCLUSIONES}

Se ha elaborado un software de predicción del aislamiento acústico de sistemas constructivos de vidrios en Matlab, mediante el modelo de Ookura\&Saito. Como datos de entrada del programa se han utilizado las constantes elásticas y el factor de pérdidas de cada elemento del sistema constructivo que, o bien se han determinado según las normativas comentadas, o bien se han obtenido de las referencias consultadas. Los resultados de pérdidas por transmisión de la configuración multicapa de vidrios obtenidos con el software de predicción se han comparado con medidas experimentales realizadas en cámara de transmisión y con datos recogidos en la bibliografía, obteniéndose un grado de ajuste aceptable con un error no mayor de $\pm 3 \mathrm{~dB}$ en el rango de frecuencias medio.

\section{AGRADECIMIENTOS}

Este trabajo ha sido financiado por el Ministerio de Ciencia y Tecnología. (MAT2003-04068) y por el Ministerio de Fomento (REF 80026/A04).

\section{BIBLIOGRAFÍA}

1. J. A. Marsh, "The Airborne Sound Insulation of Glass: Part 3", Applied Acoustics, 4, 3, 175-191, 1971.

2. UNE-EN ISO 140-1: Medición del aislamiento acústico en los edificios y de los elementos de construcción. Parte 1: Requisitos de las instalaciones del laboratorio sin transmisiones indirectas, 1997.

3. UNE-EN ISO 140-3: Medición del aislamiento acústico en los edificios y de los elementos de construcción. Parte 3: Medición en laboratorio del aislamiento acústico al ruido aéreo de los elementos de construcción, 1995.

4. UNE-EN 20140-2: Medición del aislamiento acústico en los edificios y de los elementos de edificación. Parte 2: Determinación, verificación y aplicación de los datos de precisión.

5. K. Ookura, Y. Saito, "Transmisión Loss of multiple panels containing sound absorbing materials in a random incidence field", Proceedings 1978 International Conference on Noise Control Engineering, Inter-Noise 78, San Francisco, California, May 1978.

6. A. Trochidis, A. Kalaroutis, "Sound transmission through double partitions with cavity absorption", Journal of Sound and Vibration 107, 321, 1986.

7. M. Bruneau, “Manual d'Acoustique fondamentale", Edition Hermès, 1998.

8. W. Lauriks, P. Mees, J. F. Allard, "The Acoustic Transmission Through Layered Systems", Journal of Sound and Vibration 155, 1, 125-132, 1992.

9. A. J. Price and M. J. Crocker, "Sound Transmission through Double Panels Using Statistical Energy Analysis", J. Acoust. Soc. Am. 47, 3, 683-693, 1969.
10. Panneton R., Atalla N., “Numerical Prediction Of Sound Transmission Through Finite Multilayer Systems With Poroelastic Materials", J. Acoust. Soc. Am. 100, 1 346-354, 1996.

11. UNE-EN 1748-1. Vidrio para la edificación. Productos básicos especiales. Parte 1: Vidrios borosilicatados.

12. UNE-EN 12758. Vidrio para la construcción. Acristalamiento y aislamiento al ruido aéreo. Definiciones y determinación de las propiedades.

13. UNE-EN 572-1. Vidrio para la construcción. Productos básicos de vidrio. Vidrio de silicato dsodocálcico. Parte 1: Definiciones y propiedades generales físicas y mecánicas.

14. J. Alba, J. Ramis, "Modeling of impervious layers from measurements of the sound reduction index", Applied Acoustics 64, 385, 2003.

15. J. Llinares, A, Llopis, J. Sancho, "Acústica Arquitectónica y Urbanística", Servicio de publicaciones de la Universidad Politécnica de Valencia, ISBN: 84-7721-441-7.

16. UNE-EN ISO 140-4: Medición del aislamiento acústico en los edificios y de los elementos de construcción.. Parte 4: Medición "in situ" del aislamiento al ruido aéreo entre locales.

17. M.Karjalainen, P.Antsalo, A.Mäkivirta, T.Peltonen, V.Välimäki, “Estimation of modal decay parameters from noisy response measurements, J.Audio Eng. Soc., Vol. 50, 11, Noviembre 2002.

18. K. Renji, P. S. Nair, S. Narayanan "Critical and coincidence frequencies of flat panels", Journal of Sound and Vibration 205, 1, 19-32, 1997.

19. ISO/PAS 16940:2004(E)- Glass in building - Glazing and airborne sound insulation - Measurement of the mechanical impedance of laminated glass.

20. ASTM/C 623-92 (Reapproved 2000)- Standard Test Method for Young's Modulus, Shear Modulus, and Poisson's Ratio for Glass-Ceramics by Resonance.

21. ASTM/E 756-98- Standard Test Method for Measuring Vibration-Damping Properties of Materials.

22. Y. Miki, "Acoustical properties of porous materials. Generalizations of empirical models", The Journal of the Acoustical Society of Japan, 11, 1, 25-28, 1990.

23. Y. Miki, "Acoustical properties of porous materials. Modifications of Delany \& Bazley models", The Journal of the Acoustical Society of Japan, 11, 1, 19-24, 1990.

24. J. F. Allard, Y. Champoux, “New empirical equations for sound propagation in rigid frame fibrous materials", The Journal of the Acoustical Society of America, 91, 6, 1992.

25. L. R. Dharani, J. Wei, J. Yu, R. A. Behr, P. A. Kremer, "Laminated Architectural Glass Subjected to Blast, Impact Loading", American Ceramic Cociety Bulletin, Vol. 84, No. 1, 2005.

26. J. Wei, L. R. Dharani, "Fracture mechanics of laminated glass subjected to blast loading", Theoretical and Applied Fracture Mechanics 44, 157-167, 2005.

27. A. V. Duser, A. Jagota, S. J. Bennison, «Analysis of glass/polyvinil butyral laminates subjected to uniform pressure », Journal of Engineering Mechanics, 125, 4, 435-442, april 1999.

28. R. A. Behr, J. E. Minor, H. S. Norvilla, "Structural behavior of architectural laminated glass", Journal of Structural Engineering, 119, 1, january, 202-222, 1993.

29. P. A. Du Bois, S. Kolling, W. Fassnacht, “Modelling of safety glass for crash simulation", Computational Materials Science 28, 675-683, 2003.

30. R. A. Behr, J. E. Minor, M. P. Linden, C. V. G. Vallabhan, "Laminated glass units under uniform lateral pressure", Journal of Structural Engineering, 111, 5, 1037-1050, 1985.

31. C. V. G. Vallabhan, Y. C. Das, M. Magdi, M. Asik, J. R. Bailey, “Analysis of laminated glass units", Journal of Structural Engineering, 119, 5, 1572-1585, 1993.

32. C. V. G. Vallabhan, M. Z. Asik, K. Kandil, “Analysis os structural glazing systems", Computers \& Structures, 65, 2, 231-239, 1997.

33. M. Z. Asik, C. V. G. Vallabhan, "On the convergence of nonlinear plate solutions", Computers \& Structures, 65, 2, 225-229, 1997. 